\title{
Interferometric mapping of magnetic fields: The ALMA view of the massive star forming clump W43-MM1
}

\author{
Paulo C. Cortes ${ }^{1,2}$, Josep M. Girart ${ }^{3,4}$, Charles L. H. Hull ${ }^{4,11}$, Tirupati K. Sridharan ${ }^{4}$, Fabien Louvet ${ }^{5}$, \\ Richard Plambeck ${ }^{6}$, Zhi-Yun Li ${ }^{7}$, Richard M. Crutcher ${ }^{8}$, \& Shih-Ping Lai ${ }^{9,10}$ \\ National Radio Astronomy Observatory, Charlottesville, VA 22903, USA \\ Joint ALMA Office, Alonso de Cordova 3107, Vitacura, Santiago, Chile \\ Institut de Ciències de l'Espai, (CSIC-IEEC), Campus UAB, Carrer de Can Magrans S/N, 08193 \\ Cerdanyola del Vallés, Catalonia, Spain \\ Harvard-Smithsonian Center for Astrophysics, 60 Garden St., Cambridge, MA 02138, USA \\ Departamento de Astronomia - Universidad de Chile \\ Astronomy Department $\mathcal{E}$ Radio Astronomy Laboratory, University of California, Berkeley, CA \\ 94720-3411, USA \\ Astronomy Department, University of Virginia, Charlottesville, VA 22904, USA \\ Astronomy Department, University of Illinois at Urbana-Champaign, IL 61801, USA \\ Institute of Astronomy and Department of Physics, National Tsing Hua University, Hsinchu 30013, Taiwan \\ Institute of Astronomy and Astrophysics, Academia Sinica, P.O. Box 23-141, Taipei 10617, Taiwan \\ Jansky Fellow of the National Radio Astronomy Observatory \\ pcortes@alma.cl
}

\begin{abstract}
Here we present the first results from ALMA observations of $1 \mathrm{~mm}$ polarized dust emission towards the W43-MM1 high mass star forming clump. We have detected a highly fragmented filament with source masses ranging from $14 \mathrm{M}_{\odot}$ to $312 \mathrm{M}_{\odot}$, where the largest fragment, source A, is believed to be one of the most massive in our Galaxy. We found a smooth, ordered, and detailed polarization pattern throughout the filament which we used to derived magnetic field morphologies and strengths for 12 out of the 15 fragments detected ranging from 0.2 to $9 \mathrm{mG}$. The dynamical equilibrium of each fragment was evaluated finding that all the fragments are in a super-critical state which is consistent with previously detected infalling motions towards W43-MM1. Moreover, there are indications suggesting that the field is being dragged by gravity as the whole filament is collapsing.
\end{abstract}

Subject headings: ISM: Magnetic Fields, ISM: clouds, ISM: Kinematics and dynamics 


\section{INTRODUCTION}

W43-MM1 is a large and young high mass star forming clump located within the W43 region and at 5.5 kpc from the sun (Motte et al. 2003; Zhang et al. 2014a). The clump is near $l=31^{\circ}, b=0^{\circ}$ and at an interface with an extended H II region powered by a cluster of $\mathrm{O}$ type and Wolf-Rayet stars. (Cesaroni et al. 1988; Liszt 1995; Moonev et al. 1995). W43-MM1 has been well studied in continuum from $1.3 \mathrm{~mm}$ to 70 $\mu \mathrm{m}$ (Motte et al. 2003; Bally et al. 2010). These studies identified a large sample of clumps in W43, from which W43-MM1 is the most massive with an estimated mass of $2128 \mathrm{M}_{\odot}$ and a deconvolved size of 0.09 pc (Louvet et al. 2014). Infalling motions have been detected towards W43-MM1 (Cortes et al. 2010) suggesting that the clump is undergoing gravitational collapse. Cortes \& Crutcher (2006) made interferometric observations of polarized dust emission with BIMA, finding an ordered pattern for the magnetic field and estimating an on-the-plane of the sky field strength of about $1 \mathrm{mG}$. Recent SMA results from polarized dust emission and $\mathrm{CH}_{3} \mathrm{CN}$ line emission at higher angular resolution $(\sim 0.1 \mathrm{pc}$ scales) updated the magnetic field estimate to $6 \mathrm{mG}$ also computing a mass to magnetic flux ratio of about the critical value (Sridharan et al. 2014). Additionally from the $\mathrm{CH}_{3} \mathrm{CN}$ emission, evidence for an embedded hot core of $\sim 300 \mathrm{~K}$ was found in W43-MM1 main clump. In this Letter we report the first ALMA observations of polarized dust emission towards W43-MM1. Here, section 2 report the observations, section 3 the continuum emission and source extraction, section 4 the magnetic field properties, and section 5 is the summary and discussion.

\section{OBSERVATIONS}

ALMA observations at $1 \mathrm{~mm}$ (band 6) were done on May 30th, 2015 over W43-MM1 using $\alpha=18: 47: 47.0$ and $\delta=-01: 54: 28.0$ as phase center. An array of 35 antennas was used reaching an angular resolution of $0.5^{\prime \prime}(\sim 0.01 \mathrm{pc}$ scales). The spectral configuration was set to single spectral windows per baseband in continuum mode with 64 channels giving $31.250 \mathrm{MHz}$ as the spectral resolution in full polarization mode $(X X, Y Y, Y X$, and $X Y)$. Each spectral window was centered at the standard ALMA band 6 polarization frequencies $(224.884,226.884,238.915$, and $240.915 \mathrm{GHz})$, where two successful executions were done as part of the session scheme (Remijan 2015). Calibration and imaging was done using the Common Astronomical Software Applications (CASA) version 4.5.

\subsection{Calibration and imaging in full polarization mode}

The ALMA antennas are equipped with receivers sensitive to linear polarization. After the incoming radiation has gone through the feed-horn, the wave is divided into two orthogonal components $(X$ and $Y)$ by a wave splitting device (Remijan 2015). This operation is not perfect and there is always a residual, or projection, from one polarization onto the other which is known as the instrumental polarization, or D-terms J. P. Hamaker. \& J. D. Bregman 1996). Given that an antenna uses azimuth and elevation coordinates, the frame of the sky rotates with respect to the antenna introducing an angular dependence which is parameterized by the parallactic angle. Additionally to the D-terms, the $X$ and $Y$ polarizations have different signal 
paths which introduces a relative delay between both polarizations. Also, the interferometric calibration scheme for amplitude and phase requires the use of a reference. This reference breaks the degeneracy intrinsic to the array and thus; we do not measure absolute phase values but relative ones with respect to the reference (where phases are set to zero in both polarizations). By doing this, we introduce an additional phase bandpass between the $X Y$ and $Y X$ cross correlations. To calibrate all these quantities, an ALMA polarization observation samples a strong, un-resolved, polarized source over a certain range of parallactic angle. The polarization calibrator is sampled for 5 minutes every 35 minutes or so (the precise time cadence is calculated by the online software at run time). For our observations, we obtained about $100^{\circ}$ of parallactic angle coverage for J1924-292 which was selected as polarization calibrator. Using this source, we derived solutions for the cross polarization delay, the $X Y$-phase, and the D-term 1 . These solutions were applied to W43-MM1 data also using J1751+0939 to calibrate the bandpass, J1851+0035 to calibrate the phase, and Titan to calibrate the flux. After applying the calibration tables, we imaged the data using the clean CASA task with the Briggs weighting scheme, robust number 0.5, for sidelobe robustness and the Clark deconvolution algorithm to produce the Stokes images. The final images were produced after three self-calibration iterations using a final solution interval of 90 seconds 2 .

\section{CONTINUUM EMISSION}

Figure1 1 presents the Stokes I image from W43-MM1. The continuum emission shows a fragmented filament extending from the north to the south-west. Two bright sources at the center, A and B1, completely dominate the energy budget in W43-MM1 (with integrated fluxes of $\sim 2.0$ and $0.5 \mathrm{Jy}$, which correspond to $\sim 63 \%$ of the total flux recovered), over a number of additional fragments extending to the south-west. Also, additional sources to the east and west have been detected. Comparing with the SMA results from Sridharan et al. (2014) and the PdBI $1 \mathrm{~mm}$ results from Louvet et al. (2014), the ALMA observations reproduce quite well the overall morphology of W43-MM1, but with better resolution. The noise in the ALMA map is $\sigma=0.41$ mJy beam ${ }^{-1}$ with a peak of $503 \mathrm{mJy}_{\text {beam }}^{-1}$ obtained from Gaussian fitting. We used the getsources algorithm (Men'shchikov et al. 2012) to successfully extract 14 sources from our ALMA data (see Table 1 and Figure (1). The extraction was later compared to other methods such as clumpfind (Williams et al. 1994), FellWalker, and Reinhold (Berry et al. 2007) obtaining a good agreement with the selection produced by getsources. We used the source positions and sizes derived using getsources as initial guess for a Gaussian 2D fitting (using CASA imfit algorithm) in order to derived accurate fluxes from the extracted sources 3 . Also, we kept the same source nomenclature used by Sridharan et al. (2014), but adding numbers where higher multiplicity was discovered with respect to the SMA map. Using the standard procedure to calculate masses

\footnotetext{
${ }^{1}$ More details about the calibration procedure can be found in the 3c286 ALMA science verification data casa-guide at https://casaguides.nrao.edu/index.php/3C286_Band6Pol_Calibration_for_CASA_4.3

${ }^{2}$ In principle the solution interval when self-calibrating, is calculated based on the the sensitivity. However, we noticed that using smaller solution intervals increased the image noise and thus, we stopped the iteration at $90 \mathrm{~s}$

${ }^{3}$ We found that getsources tend to underestimate the recovered fluxes from our data
} 
from dust emission (Hildebrand 1983) we computed masses for all 15 sources in our catalog assuming a dust opacity of $\kappa_{1.3 \mathrm{~mm}}=0.01 \mathrm{~cm}^{2} \mathrm{~g}^{-1}$ (Ossenkopf \& Henning 1994), a gas to dust ratio of 1:100, and a dust temperature of $T_{\text {dust }}=25 \mathrm{~K}$ (Bally et al. 2010), with the exception of the hot core, source A, where we used a range between $70<T_{\text {dust }}<150 \mathrm{~K}$. Although the SMA detected $\mathrm{CH}_{3} \mathrm{CN}$ emission towards B1 and $\mathrm{C}$, it was unresolved and not sufficient to derived temperatures; hence, we used $T_{\text {dust }}=25 \mathrm{~K}$ for these sources. Herpin et al. (2012) modeled an SED and derived a temperature profile for source A using all the publicly available data on W43-MM1 to date. Their model suggests a temperature of $\sim 150 \mathrm{~K}$ at $2500 \mathrm{AU}$ distance $\left(0.5^{\prime \prime}\right.$ radial; $1^{\prime \prime}$ size) and $\sim 70 \mathrm{~K}$ at about $8000 \mathrm{AU}$ distance $\left(0.7^{\prime \prime}\right.$ radial; $1.4^{\prime \prime}$ size $)$, which are the length-scales sampled by ALMA. However if larger spatial scales than our source size are considered, the temperature might be lower and in the order of $30 \mathrm{~K}$ (as suggested by Bally et al. 2010). These scales $\left(>10^{\prime \prime}\right.$ ) are consistent with Herschel primary beam at $160 \mu \mathrm{m}$ and, off course, larger than our deconvolved source sizes and synthesized beam. Therefore and using this temperature range, our derived mass for source A is between 312 and $146 \mathrm{M}_{\odot}$ (with the exception of the $30 \mathrm{~K}$ temperature derived mass of $728 \mathrm{M}_{\odot}$ ). These estimates put source A below other massive clumps such as the SDC335-MM1 mass estimate of $545 \mathrm{M}_{\odot}$ (Peretto et al. 2013) and G31.41+0.31 with a mass estimate of $577 \mathrm{M}_{\odot}$ (Girart et al. 2009) 4 . However, these mass estimates where derived from observations sampling larger length scales than our ALMA W43 observations. The deconvolved size of SDC335-MM1 is about 0.054 which is 2 times the size of source A or 4 times the area. A simple estimate assuming a $r^{-2}$ density profile, will give about $272 \mathrm{M}_{\odot}$ per source A size for SDC335-MM1 and about $205 \mathrm{M}_{\odot}$ per source A size for G31.41+0.31.

\section{THE MAGNETIC FIELD MORPHOLOGY}

The polarized emission from W43-MM1 shows fractional polarization levels between $0.03 \%$ to $22 \%$ where the lowest values are seen over the peaks in Stokes I, which corresponds to the well known polarization-hole (Hull et al. 2014, and references therein). Assuming perfect grain alignment, the magnetic field morphology onto the plane of the sky is inferred from the polarized emission by rotating the electric vector position angle (EVPA) by $90^{\circ}$ and shown in Figure 2. The field morphology shows a smooth and ordered pattern over the filament on angular scales $<15^{\prime \prime} 5$, where ordered magnetic fields are found in massive dense cores when observed at similar spatial scales, as it has been shown from a relatively large sample by Zhang et al. (2014b). We found overall agreement with the SMA results, but now given our higher resolution and sensitivity, we can see the field morphology in greater detail. In fact for source A, there is a $\sim 90^{\circ}$ change in orientation to the west of the clump, where there is also a decrease in polarized intensity, and towards D1, the field changes about $90^{\circ}$ in orientation with respect to the main filament. For analysis, we divided the filament into four regions according to the boundaries in the field pattern. Although, the field morphology is continuous from

\footnotetext{
${ }^{4}$ Note that in this massive core a magnetic field strength of $10 \mathrm{mG}$ was derived using $\mathrm{n}\left(\mathrm{H}_{2}\right)=3 \times 10^{6} \mathrm{~cm}^{-3}$

${ }^{5}$ The ALMA polarization accuracy of $0.1 \%$ in fractional polarization is only guaranteed within $1 / 3$ of FWHM, which in our data this corresponds to $\sim 10^{\prime \prime}$. However, recent results from ALMA commissioning (Cortes 2015) showed that the systematic error within $-3 \mathrm{~dB}$ level $\left(\sim 15^{\prime \prime}\right)$ of the primary beam is less than $0.5 \%$ in band 6 , which is larger than the size of the W43-MM1 filament. In fact, our observations are consistent to the SMA data beyond the 1/3 of FWHM or $10^{\prime \prime}$ limit.
} 
A to B1, we set the boundary to the north of B2 where we plotted the lowest contour in the polarized intensity. We did that in order to analyze the field locally to source A and sources B1, B2, B3, B4, and E.

To understand the dynamical importance of the magnetic field over W43-MM1, we estimated the strength of the field using the Chandrasekhar \& Fermi technique (here after CF Chandrasekhar \& Fermi 1953) as follows from Crutcher et al. (2004).

$$
B_{\mathrm{pos}}=9.3 \frac{\sqrt{n_{\mathrm{H}_{2}}} \Delta V}{\delta \phi}
$$

where $n_{\mathrm{H}_{2}}$ is the molecular Hydrogen number density calculated as an average over the region sampled by the polarized dust emission, $\Delta V$ is the FWHM from a line tracing the gas motions in W43 which in this case was taken to be $3.0 \mathrm{~km} / \mathrm{s}$ from $\mathrm{H}^{13} \mathrm{CO}^{+}(4-3)$ and $\mathrm{DCO}^{+}(5-4)$ single dish emission (Cortes et al. 2010; Cortes 2011), and $\delta \phi$ is the EVPA dispersion, which we calculated as the standard deviation of the EVPAs for each region. Thus, the derived field strengths are local to all the sources in a particular region. Table 2 summarizes our polarization results with the corresponding estimations for the field.

The usage of the CF method have been debated given the small angle dispersion approximation required and the assumption of energy equipartition. From Table 2 we see that 2 out of 4 of our regions have EVPA dispersions larger than the $25^{\circ}$ limit suggested by Ostriker et al. (2001). Given this, we included modified versions of the CF method (Heitsch et al. 2001; Falceta-Goncalves et al. 2008), to calculate the field strength on each region independently. Heitsch et al. (2001) attempt to address the limitation of the small angle approximation by replacing $\delta \phi$ by $\delta \tan (\phi)$ which is calculated locally and by adding a geometric correction to avoid underestimating the field in the super-Alfvenic case. In contrast, Falceta-Gonçalves et al. (2008) assumed that the field perturbation is a global property and thus, they replaced $\delta \phi$ by $\tan (\delta \phi) \sim \delta B / B_{s k y}$ in the denominator of equation 11. By using the 3 versions of the CF method, we obtained estimations of the magnetic field between 0.2 to $7 \mathrm{mG}$ for our 4 regions, where for source $\mathrm{A}$ we obtained values between 1 and $4 \mathrm{mG}$. Crutcher (2012) plotted the most up-to-date field profile from Zeeman measurements. Figures 6 and 7 in that work, suggests that our estimates for $B_{\text {pos }}$ are consistent with the curve if we extrapolate the profile as, unfortunately, the plot lacks field values for the $10^{25} \mathrm{~cm}^{-2}$ range in column density.

\section{SUMMARY AND DISCUSSION}

The ALMA results on W43_MM1 suggest that we are seeing a highly fragmented filament where the emission is dominated by a single clump, source A. Indeed, from Table 1 we see that the bulk of fragments (13) have fluxes between 32 and $200 \mathrm{mJy}$; while source A alone is about $2 \mathrm{Jy}$. The high degree of fragmentation seen in the ALMA data pose the question about the gravitational stability of these sources. Although we do not have high resolution line data to address the kinematics and determine if these sources are selfgravitating, the infalling motions detected by Cortes et al. (2010) (covering an area of $48^{\prime \prime} \times 48^{\prime \prime}$ ) are likely showing accretion from larger scales onto the whole filament and suggesting gravitational collapse. To test the gravitational stability of each fragment, we calculated the thermal Jeans length as $\lambda_{J}=c_{s}(\pi / G \rho)^{1 / 2}$, 
where $c_{s}=\sqrt{K T / m_{\mathrm{H}_{2}}}$ the sound speed, $\rho$ is the volume density, and $G$ is the gravitational constant. We found that our deconvolved sizes are larger than the estimated Jeans lengths by roughly an order of magnitude for most fragments. Thus, we cannot conclude what fraction of these fragments are gravitationally unstable from these data alone. Interestingly is the case of source A which has been determined to be gravitationally bound (Louvet et al. 2014; Cortes et al. 2010, and references therein) but its deconvolved size is between a factor of 10 and 6 larger than its Jeans length. If the Jeans length suggests further fragmentation, it is possible that source A has a larger multiplicity of fragments which requires higher angular resolution to resolve.

Using the derived magnetic field estimations, we can compute the mass to magnetic flux ratio for all our sources using our $\mathrm{B}_{\text {pos }}$ estimations. We do this following Crutcher et al. (2004) as,

$$
\lambda_{B}=7.6 \times 10^{-21} \frac{N\left(\mathrm{H}_{2}\right)}{3 \mathrm{~B}_{\mathrm{pos}}}
$$

where $\mathrm{N}\left(\mathrm{H}_{2}\right)$ is molecular hydrogen column density in $\mathrm{cm}^{-2}$ calculated for each source independently, $\mathrm{B}_{\text {pos }}$ is the magnetic field strength in $\mu G$ assumed to be unique for a given region, and factor of 3 in the denominator corresponds to an statistical geometrical correction (Crutcher et al. 2004). This statistical geometrical factor of 3 is for a uniform density slab and will be smaller than 3 for more physically realistic cases, such as a centrally condensed disk. We found highly super-critical values for all fragments in the filament (see Table 2), showing that the field is not strong enough to support them against gravity. Also, it is worth noting that polarized emission decreases significantly towards the dust peaks in W43-MM1, which has already been observed towards DR21(OH) by the SMA (Girart et al. 2013). This is particularly evident towards source A where the polarized intensity emission goes below the $5 \sigma$ level close the dust peak. Additionally, the $\mathrm{B}_{\text {pos }}$ morphology in source A seems to indicate the field weakness as the lines appear to be bent towards the dust peak. This additional evidence, suggests that gravity is pulling the field lines as the gas is infalling due to gravitational collapse. However, what fraction of these fragments are bound by gravity is still open; and thus, further fragmentation cannot be ruled out.

Here, we have presented ALMA polarized dust emission observations of W43-MM1. We have found a fragmented filament threaded by a smooth, ordered, and highly detailed magnetic field. We have derived mass estimates for 15 fragments extracted from the continuum map, showing that source A dominates the flux distribution with a $51 \%$ of the total flux. Using temperature modeling by others, we derived a mass range for source A between 146 and $312 \mathrm{M}_{\odot}$ under the length-scales sampled by ALMA. However, it is not clear if further fragmentation is ongoing inside source A and only higher resolution ALMA observations can reveal this. We have derived magnetic field strengths for all fragments with sufficient polarization data and found field estimations in range of $0.2-7 \mathrm{mG}$ using 3 versions of the CF method. Derivation of the mass to magnetic flux ratio indicate that the fragments are super-critical suggesting that the field is dominated by gravity at this stage of the W43-MM1 evolution.

The National Radio Astronomy Observatory is a facility of the National Science Foundation operated under 


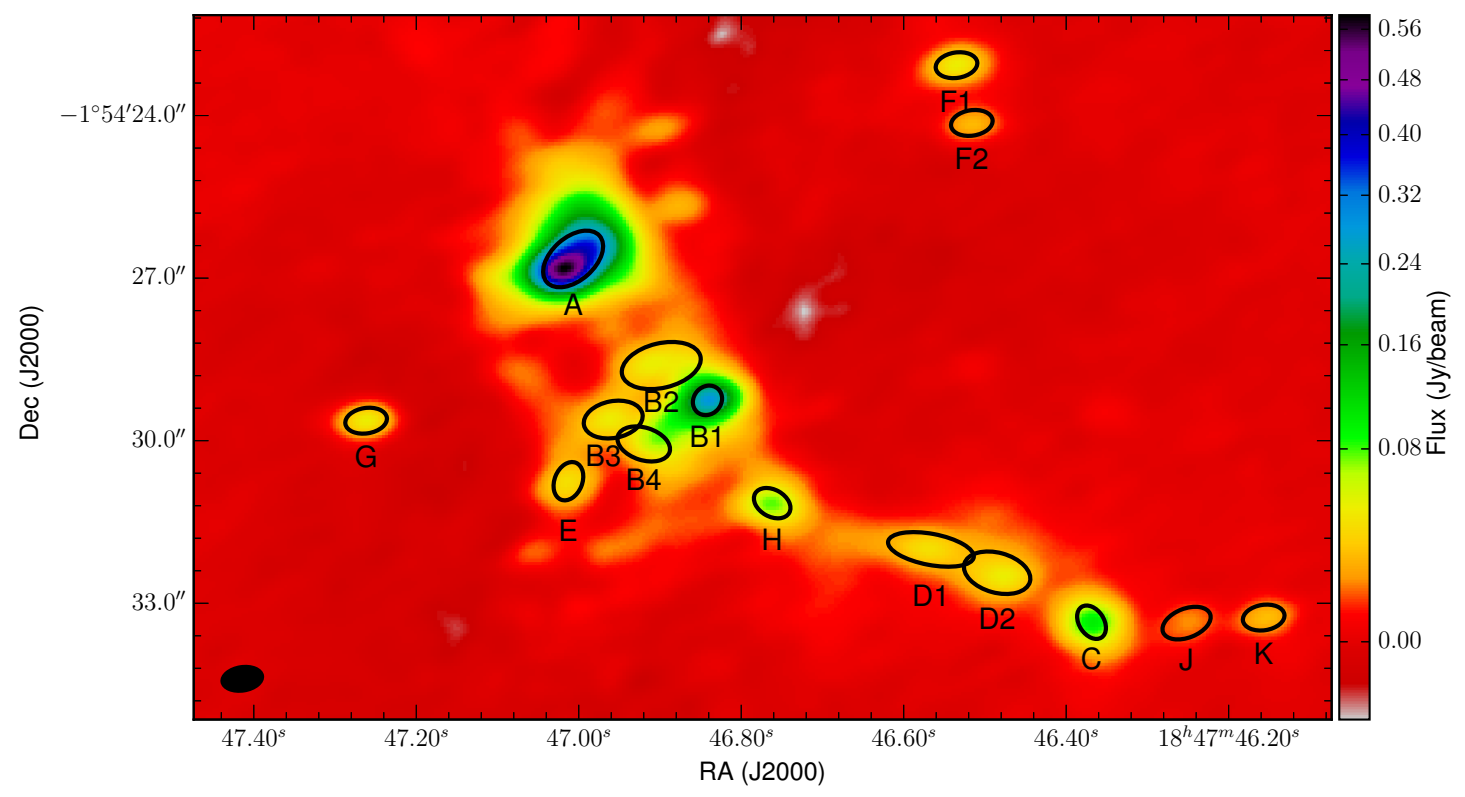

Fig. 1. - The Figure shows the Stokes I emission from the W43_MM1 clump indicated by the color-scale in Jy beam ${ }^{-1}$. Overlaid are the sources extracted as ellipses, in black, representing the deconvolved sized obtained from the Gaussian fits. With an rms noise of $\sigma=0.41 \mathrm{mJy}^{\mathrm{beam}}{ }^{-1}$, the dynamic range of the self-calibrated image is 1220 . 


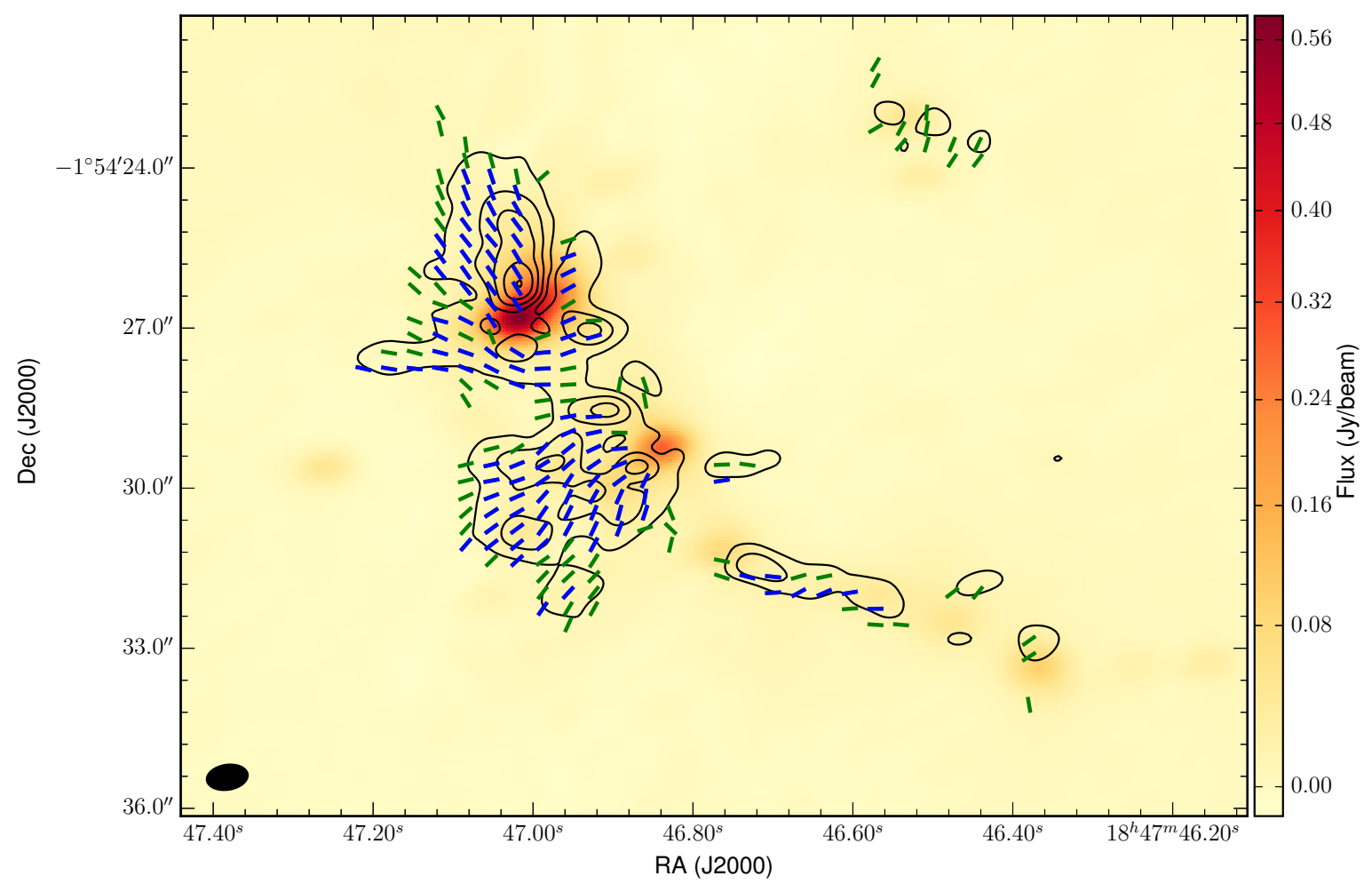

Fig. 2.- The Figure shows the magnetic field morphology over W43-MM1. The Stokes I emission is shown as color scale, the dust, rician debiased, polarized intensity is shown in contours of $0.55,1.3,2.0,2.7$, and $3.5 \mathrm{mJy}$ beam $^{-1}$. The magnetic field morphology is shows as pseudo-vectors at a significance of $3 \sigma$ in green and $5 \sigma$ in blue, where $\sigma=93 \mu \mathrm{Jy}_{\text {beam }}^{-1}$ corresponds to the noise in the polarized intensity image. The length of each pseudo-vectors is normalized. Also, each pseudo-vector is plotted every half-beam, i.e. in steps of 8 and 4 pixels, where the beam is $13 \times 7$ pixels. 
cooperative agreement by Associated Universities, Inc.

P.C.C. would like to thank Ed Fomalont for very helpful and enlighten discussion about polarization data analysis.

J.M.G. acknowledges support from MICINN AYA2014-57369-C3-P and the MECD PRX15/00435 grants (Spain).

Z.Y.L. is supported in part by NASA NNX14AB38G and NSF AST-1313083.

S.P.L. thanks the support of the Ministry of Science and Technology (MoST) of Taiwan through Grants NSC 98-2112-M-007-007-MY3, NSC 101-2119-M-007-004, and MoST 102-2119-M-007-004- MY3.

This research made use of APLpy, an open-source plotting package for Python hosted at http://aplpy.github.com

\section{REFERENCES}

Bally, J., Anderson, L. D., Battersby, C., Calzoletti, L., Digiorgio, A. M., Faustini, F., Ginsburg, A., Li, J. Z., Nguyen Luong, Q., Molinari, S., Motte, F., Pestalozzi, M., Plume, R., Rodon, J., Schilke, P., Schlingman, W., Schneider-Bontemps, N., Shirley, Y., Stringfellow, G. S., Testi, L., Traficante, A., Veneziani, M., \& Zavagno, A. 2010, A\&A, 518, L90

Berry, D. S., Reinhold, K., Jenness, T., \& Economou, F. 2007, in Astronomical Society of the Pacific Conference Series, Vol. 376, Astronomical Data Analysis Software and Systems XVI, ed. R. A. Shaw, F. Hill, \& D. J. Bell, 425

Cesaroni, R., Palagi, F., Felli, M., Catarzi, M., Comoretto, G., di Francos, Giovanardi, C., \& Palla, F. 1988, A\&AS, 76, 445

Chandrasekhar, S. \& Fermi, E. 1953, ApJ, 118, 113

Cortes, P. \& Crutcher, R. M. 2006, ApJ, 639, 965

Cortes, P. C. 2011, ApJ, 743, 194

Cortes, P. C., Parra, R., Cortes, J. R., \& Hardy, E. 2010, A\&A, 519, A35

Cortes, P. C. e. a. 2015, ALMA Technical note 10: EOC ALMA Polarization commissioning results

Crutcher, R. M., Nutter, D. J., Ward-Thompson, D., \& Kirk, J. M. 2004, ApJ, 600, 279

Crutcher, R. M. 2012, ARA\&A, 50, 29

Falceta-Gonçalves, D., Lazarian, A., \& Kowal, G. 2008, ApJ, 679, 537

Frau, P., Galli, D., \& Girart, J. M. 2011, A\&A, 535, A44

Girart, J. M., Beltrán, M. T., Zhang, Q., Rao, R., \& Estalella, R. 2009, Science, 324, 1408 
Girart, J. M., Frau, P., Zhang, Q., Koch, P. M., Qiu, K., Tang, Y.-W., Lai, S.-P., \& Ho, P. T. P. 2013, ApJ, 772,69

Heitsch, F., Zweibel, E. G., Mac Low, M.-M., Li, P., \& Norman, M. L. 2001, ApJ, 561, 800

Herpin, F., Chavarría, L., van der Tak, F., Wyrowski, F., van Dishoeck, E. F., Jacq, T., Braine, J., Baudry, A., Bontemps, S., \& Kristensen, L. 2012, A\&A, 542, A76

Hildebrand, R. H. 1983, QJRAS, 24, 267

Hull, C. L. H., Plambeck, R. L., Kwon, W., Bower, G. C., Carpenter, J. M., Crutcher, R. M., Fiege, J. D., Franzmann, E., Hakobian, N. S., Heiles, C., Houde, M., Hughes, A. M., Lamb, J. W., Looney, L. W., Marrone, D. P., Matthews, B. C., Pillai, T., Pound, M. W., Rahman, N., Sandell, G., Stephens, I. W., Tobin, J. J., Vaillancourt, J. E., Volgenau, N. H., \& Wright, M. C. H. 2014, ApJS, 213, 13

J. P. Hamaker,, R. \& J. D. Bregman. 1996, Astron. Astrophys. Suppl. Ser., 117, 149

Liszt, H. S. 1995, AJ, 109, 1204

Louvet, F., Motte, F., Hennebelle, P., Maury, A., Bonnell, I., Bontemps, S., Gusdorf, A., Hill, T., Gueth, F., Peretto, N., Duarte-Cabral, A., Stephan, G., Schilke, P., Csengeri, T., Nguyen Luong, Q., \& Lis, D. C. 2014, A\&A, 570, A15

Men'shchikov, A., André, P., Didelon, P., Motte, F., Hennemann, M., \& Schneider, N. 2012, A\&A, 542, A81

Mooney, T., Sievers, A., Mezger, P. G., Solomon, P. M., Kreysa, E., Haslam, C. G. T., \& Lemke, R. 1995, A\&A, 299, 869

Motte, F., Schilke, P., \& Lis, D. C. 2003, ApJ, 582, 277

Ossenkopf, V. \& Henning, T. 1994, A\&A, 291, 943

Ostriker, E. C., Stone, J. M., \& Gammie, C. F. 2001, ApJ, 546, 980

Peretto, N., Fuller, G. A., Duarte-Cabral, A., Avison, A., Hennebelle, P., Pineda, J. E., André, P., Bontemps, S., Motte, F., Schneider, N., \& Molinari, S. 2013, A\&A, 555, A112

Remijan, A. e. a. 2015, ALMA Cycle 3 Technical Handbook

Sridharan, T. K., Rao, R., Qiu, K., Cortes, P., Li, H., Pillai, T., Patel, N. A., \& Zhang, Q. 2014, ApJ, 783, L31

Williams, J. P., de Geus, E. J., \& Blitz, L. 1994, ApJ, 428, 693

Zhang, B., Moscadelli, L., Sato, M., Reid, M. J., Menten, K. M., Zheng, X. W., Brunthaler, A., Dame, T. M., Xu, Y., \& Immer, K. 2014a, ApJ, 781, 89 
Zhang, Q., Qiu, K., Girart, J. M., (Baobab Liu, H., Tang, Y.-W., Koch, P. M., Li, Z.-Y., Keto, E., Ho, P. T. P., Rao, R., Lai, S.-P., Ching, T.-C., Frau, P., Chen, H.-H., Li, H.-B., Padovani, M., Bontemps, S., Csengeri, T., \& Juárez, C. 2014b, ApJ, 792, 116 
Table 1. Continuum Sources

\begin{tabular}{cccccccccccc}
\hline \hline ID & $\begin{array}{c}\text { R.A. } \\
{[\mathrm{J} 2000]}\end{array}$ & $\begin{array}{c}\text { DEC } \\
{[\mathrm{J} 2000]}\end{array}$ & $\begin{array}{c}\text { Peak }^{\mathrm{a}} \\
{\left[\mathrm{mJy} \mathrm{beam}^{-1}\right]}\end{array}$ & $\begin{array}{c}\text { Flux } \\
{[\mathrm{mJy}]}\end{array}$ & $\begin{array}{c}\text { Major }^{\mathrm{b}} \\
{[\operatorname{arcsec}]}\end{array}$ & $\begin{array}{c}\text { Minor }^{\mathrm{b}} \\
{[\operatorname{arcse}]}\end{array}$ & $\begin{array}{c}\text { P.A. } \\
{[\mathrm{deg}]}\end{array}$ & $\begin{array}{c}\mathrm{n}^{\mathrm{c}, d} \\
{\left[10^{7} \mathrm{~cm}^{-3}\right]}\end{array}$ & $\begin{array}{c}\text { Mass }^{\mathrm{c}} \\
{\left[\mathrm{M}_{\odot}\right]}\end{array}$ & $\begin{array}{c}\text { Size }^{\mathrm{e}} \\
{[\mathrm{mpc}]}\end{array}$ & $\begin{array}{c}\lambda_{J}^{\mathrm{c}} \\
{[\mathrm{mpc}]}\end{array}$ \\
\hline A & $18: 47: 47.0$ & $-01: 54: 26.7$ & $503.1 \pm 26$ & 1988.2 & 1.30 & 0.80 & 131.4 & $38,81.3,190$ & $146,312,729$ & 27.09 & $5,3.4,2.2$ \\
B1 & $18: 47: 46.8$ & $-01: 54: 29.3$ & $271.4 \pm 15$ & 504.1 & 0.58 & 0.51 & 138 & 384.0 & 22 & 14.4 & 1.5 \\
C & $18: 47: 46.4$ & $-01: 54: 33.4$ & $95.2 \pm 3$ & 190.5 & 0.67 & 0.46 & 33 & 133.9 & 84 & 14.8 & 2.6 \\
H & $18: 47: 46.8$ & $-01: 54: 31.2$ & $73.5 \pm 4$ & 151.9 & 0.72 & 0.50 & 60 & 85.0 & 67 & 16.0 & 3.3 \\
B4 & $18: 47: 46.9$ & $-01: 54: 30.1$ & $49.8 \pm 8$ & 121.1 & 1.00 & 0.60 & 72 & 31.3 & 53 & 20.7 & 5.4 \\
G & $18: 47: 47.3$ & $-01: 54: 29.6$ & $48.1 \pm 2$ & 48.4 & 0.77 & 0.47 & -81 & 26.6 & 21 & 16.1 & 5.9 \\
B2 & $18: 47: 46.9$ & $-01: 54: 28.6$ & $46.1 \pm 5$ & 201.3 & 1.48 & 0.82 & 103 & 17.9 & 89 & 29.5 & 7.1 \\
F1 & $18: 47: 46.5$ & $-01: 54: 23.1$ & $44.7 \pm 1$ & 71.9 & 0.77 & 0.47 & -81 & 39.4 & 32 & 16.1 & 4.8 \\
B3 & $18: 47: 47.0$ & $-01: 54: 29.6$ & $43.7 \pm 4$ & 133.3 & 1.10 & 0.68 & 101 & 24.9 & 59 & 23.0 & 6.1 \\
D2 & $18: 47: 46.5$ & $-01: 54: 32.4$ & $42.6 \pm 4$ & 153.9 & 1.26 & 0.74 & 75 & 20.8 & 68 & 25.7 & 6.6 \\
E & $18: 47: 47.0$ & $-01: 54: 30.8$ & $38.6 \pm 1$ & 84.0 & 0.73 & 0.51 & 158 & 43.8 & 37 & 16.3 & 4.6 \\
D1 & $18: 47: 46.6$ & $-01: 54: 32.0$ & $36.7 \pm 2$ & 137.1 & 1.61 & 0.58 & 79 & 18.5 & 60 & 25.7 & 7.0 \\
K & $18: 47: 46.2$ & $-01: 54: 33.3$ & $25.3 \pm 1$ & 40.4 & 0.77 & 0.47 & -81 & 22.2 & 18 & 16.1 & 6.4 \\
F2 & $18: 47: 46.5$ & $-01: 54: 24.1$ & $24.3 \pm 2$ & 32.4 & 0.77 & 0.47 & -81 & 17.8 & 14 & 16.1 & 7.2 \\
J & $18: 47: 46.3$ & $-01: 54: 33.4$ & $15.6 \pm 1$ & 37.2 & 0.93 & 0.54 & 111 & 12.7 & 16 & 18.8 & 8.5 \\
\hline
\end{tabular}

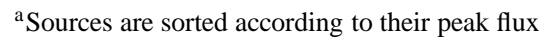

${ }^{b}$ Deconvolved major and minor axes are obtained from the Gaussian fitting procedure.

${ }^{\mathrm{c}}$ For the hot core (source A), we are showing calculations for a $T_{\text {dust }}=30,70$, and $150 \mathrm{~K}$

${ }^{\mathrm{d}}$ Volume number densities were calculated assuming spherical geometry.

${ }^{\mathrm{e}}$ The fragment size is calculated as $\mathrm{d}=5500^{*} \sqrt{b_{m a j} \times b_{\min }}[\mathrm{pc}]$. 
Table 2. Polarization data and magnetic field estimations onto the plane of the sky. All parameters are dervied assuming a temperature of $25 \mathrm{~K}$ with the exception of source A, where we are showing values for $T_{\text {dust }}=70$ and $150 \mathrm{~K}$. The polarization statistics are calculated from the $5 \sigma$ data

\begin{tabular}{|c|c|c|c|c|c|c|c|c|c|c|c|c|c|c|}
\hline Source & Region & $\begin{array}{c}\mathrm{n}_{\mathrm{r}}^{\mathrm{a}} \\
{\left[10^{7} \mathrm{~cm}^{-3}\right]}\end{array}$ & $\begin{array}{c}\mathrm{N}^{\mathrm{b}} \\
{\left[10^{24} \mathrm{~cm}^{-2}\right]}\end{array}$ & $\begin{array}{c}<\phi>^{\mathrm{c}} \\
{\left[{ }^{\circ}\right]}\end{array}$ & $\begin{array}{l}\delta \phi^{\mathrm{c}} \\
{\left[{ }^{\circ}\right]}\end{array}$ & $\begin{array}{l}\mathrm{F}_{\min }{ }^{\mathrm{c}} \\
{[\%]}\end{array}$ & $\begin{array}{c}\mathrm{F}_{\max }^{\mathrm{c}} \\
{[\%]}\end{array}$ & $\begin{array}{c}<\mathrm{F}>^{\mathrm{c}} \\
{[\%]}\end{array}$ & $\begin{array}{r}\mathrm{B}_{1}{ }^{\mathrm{d}} \\
{[\mathrm{mG}]}\end{array}$ & $\begin{array}{c}\mathrm{B}_{2}{ }^{\mathrm{e}} \\
{[\mathrm{mG}]}\end{array}$ & $\begin{array}{c}\mathrm{B}_{3}{ }^{\mathrm{f}} \\
{[\mathrm{mG}]}\end{array}$ & $\lambda_{\mathrm{B}_{1}}^{\mathrm{g}, b}$ & $\lambda_{\mathrm{B}_{2}}^{\mathrm{h}, b}$ & $\lambda_{\mathrm{B}_{3}}{ }^{\mathrm{i}, b}$ \\
\hline A & 1 & 1.4 & $68.0-31.7$ & -30.5 & 36.2 & 0.41 & 13.9 & 4.9 & 3 & 4 & 1 & $59-27$ & $40-19$ & $145-68$ \\
\hline B1 & 2 & 1.5 & 170.7 & 42.5 & 21.9 & 0.46 & 19.6 & 6.2 & 5 & 8 & 2 & 89 & 55 & 225 \\
\hline $\mathrm{E}$ & 2 & 1.5 & 22.1 & 42.5 & 21.9 & 0.46 & 19.6 & 6.2 & 5 & 8 & 2 & 12 & 7 & 29 \\
\hline B2 & 2 & 1.5 & 16.3 & 42.5 & 21.9 & 0.46 & 19.6 & 6.2 & 5 & 8 & 2 & 9 & 5 & 21 \\
\hline B3 & 2 & 1.5 & 17.7 & 42.5 & 21.9 & 0.46 & 19.6 & 6.2 & 5 & 8 & 2 & 9 & 6 & 23 \\
\hline B4 & 2 & 1.5 & 20.0 & 42.5 & 21.9 & 0.46 & 19.6 & 6.2 & 5 & 8 & 2 & 10 & 6 & 26 \\
\hline $\mathrm{C}$ & 3 & 1.1 & 61.2 & 12.8 & 49.7 & 0.87 & 9.4 & 4.1 & 2 & 2 & 0.2 & 84 & 67 & 701 \\
\hline $\mathrm{H}$ & 3 & 1.1 & 41.9 & 12.8 & 49.7 & 0.87 & 9.4 & 4.1 & 2 & 2 & 0.2 & 57 & 46 & 480 \\
\hline D2 & 3 & 1.1 & 16.4 & 12.8 & 49.7 & 0.87 & 9.4 & 4.1 & 2 & 2 & 0.2 & 22 & 18 & 189 \\
\hline D1 & 3 & 1.1 & 14.7 & 12.8 & 49.7 & 0.87 & 9.4 & 4.1 & 2 & 2 & 0.2 & 20 & 16 & 168 \\
\hline $\mathrm{F} 1$ & 4 & 0.4 & 19.6 & 54.3 & 22.0 & 2.30 & 22.4 & 6.8 & 3 & 4 & 1 & 19 & 12 & 53 \\
\hline $\mathrm{F} 2$ & 4 & 0.4 & 8.8 & 54.3 & 22.0 & 2.30 & 22.4 & 6.8 & 3 & 4 & 1 & 8 & 5 & 24 \\
\hline
\end{tabular}

${ }^{a}$ The number density used to estimate $\mathrm{B}_{\text {pos }}$, calculated from the Stokes I emission across the entire region

${ }^{b}$ For the hot core, the estimations are calculated using a temperature range of 70 and $150 \mathrm{~K}$

${ }^{\mathrm{c}}$ Here $\langle\phi\rangle$ is the average EVPA, $\delta \phi$ is the EVPA dispersion (calculated using circular statistics), $\mathrm{F}_{\min }$ is the minumum fractional polarization, $\mathrm{F}_{\text {max }}$ is the maximun fractional polarization, and $<\mathrm{F}>$ is average fractional polarization value. All values are computed for the region indicated in column 2.

${ }^{\mathrm{d}}$ Estimations of the magnetic field, in the plane of the sky, done with the original CF method (see equation 1 in the text)

${ }^{e}$ Estimations of the magnetic field, in the plane of the sky, done using the corrections implemented by Falceta-Gonçalves et al. (2008) equation 9

${ }^{\mathrm{f}}$ Estimations of the magnetic fieldin the plane of the sky, done using the corrections implemented by Heitsch et al. (2001) equation 12

${ }^{g}$ Mass to magnetic flux estimate using field strength estimate $B_{1}$

${ }^{h}$ Mass to magnetic flux estimate using field strength estimate $B_{2}$

${ }^{\mathrm{i}}$ Mass to magnetic flux estimate using field strength estimate $\mathrm{B}_{3}$ 